\title{
Strömgren photometry and medium-resolution spectroscopy of some $\delta$ Scuti and $\gamma$ Doradus stars in the Kepler field
}

\author{
L. Fox-Machado \\ Instituto de Astronomía, Universidad Nacional Autónoma de México \\ email: lfox@astrosen.unam.mx
}

\begin{abstract}
We have obtained CCD photometry and medium-resolution spectroscopy of a number of $\delta$ Scuti and $\gamma$ Doradus stars in the Kepler field-of-view as part of the ground-based observational efforts to support the Kepler space mission. In this work we present the preliminary results of these observations.
\end{abstract}

Keywords. stars: variables: $\delta$ Sct, stars: variables: $\gamma$ Dor

\section{Introduction}

The Kepler space mission (Borucki et al. 2010) was successfully launched in March 2009 and since then it has been monitoring a huge number of stars in a region of 105 square degrees located between the constellations of Cygnus and Lyra. Although the main scientific goal of the mission is to discover Earth-sized planets, the high precision photometry provided by the Kepler satellite gives a unique opportunity to study the pulsational variability of thousands of stars across the H-R diagram in details by means of asteroseismic methods (Aerts et al. 2010). As is well known, asteroseismic studies require accurate and precise atmospheric parameters of the stars to produce reliable results. Since the precision of the physical parameters like effective temperature, gravity and metallicity available in the Kepler Input Catalog (KIC, Latham et al. 2005) is generally too low for asteroseismic modelling, to best exploit the Kepler data additional multi-colour and spectroscopic information is needed. In the framework of the Kepler Asteroseismic Science Consortium (KASC, http://astro.phys.au.dk/KASC/) several ground-based observational efforts have been undertaken to derive physical parameters of the Kepler stars with high precision (e.g. Uytterhoeven et al. 2011, Molenda-Żakowicz et al. 2011). This paper describes our observational efforts at the Observatorio Astronómico Nacional at San Pedro Mártir (OAN-SPM) in Baja California, Mexico to derive the physical parameters of several $\delta$ Scuti and $\gamma$ Doradus stars in the Kepler field.

\section{Observations, data reduction and conclusion}

The CCD observations of $74 \delta$ Scuti and $\gamma$ Doradus stars in the Kepler field have been made with the 0.84-m f/15 Ritchey-Chrétien telescope at OAN-SPM, during six consecutive nights, from 2012 June 21 to June 26. The telescope hosted the filter-wheel 'Mexman' with the ESOPO (E2V) CCD camera, which has a $2048 \times 4608$ pixel array, with a pixel size of $15 \times 15 \mu \mathrm{m}^{2}$. The typical field-of-view with this configuration is $8^{\prime} \times$ $8^{\prime}$. The observations were taken with Strömgren uvby and $\mathrm{H} \beta$ filters to take advantage of the Strömgren-Crawford photometric system in deriving physical parameters of the 


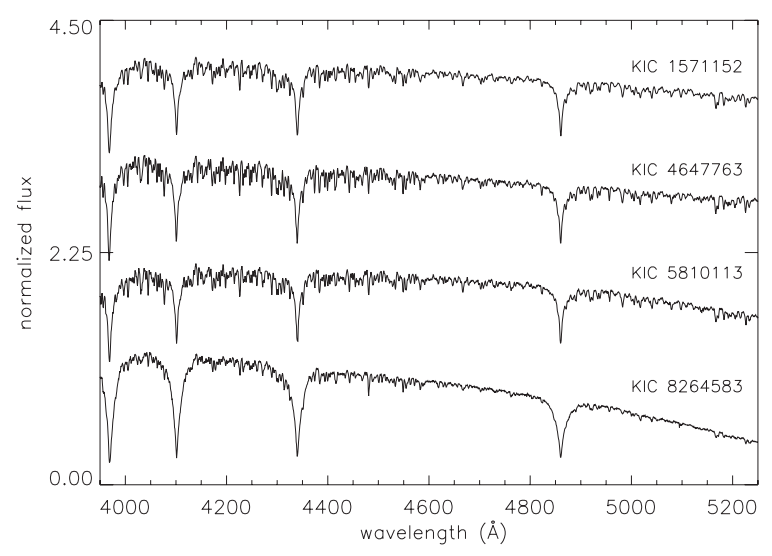

Figure 1. Reduced spectra of some Kepler targets.

stars. A set of standard stars from well observed open clusters (e.g. Peña et al. 2011) was also observed each night to transform instrumental observations onto the standard system and to correct for atmospheric extinction. The usual calibration procedures for CCD photometry have been carried out during our observing run. Sky flat fields, bias and dark exposures were taken every night. The data reduction of this CCD photometry has been carried out with the usual techniques and IRAF packages. The instrumental magnitudes and colours, once corrected for atmospheric extinction were transformed to the standard system. In this way, we have obtained the standard Strömgren indices not only of the $74 \delta$ Scuti and $\gamma$ Doradus target stars, but also of all the stars brighter than $V \sim 15$ mag located within each observed field.

The spectroscopic observations were conducted at the $2.12-\mathrm{m}$ telescope of the OANSPM observatory during several short runs between 2010 and 2012. We used the same equipment as explained in Fox Machado et al. (2010). In particular, we used the Boller \& Chivens spectrograph installed in the Cassegrain focus of the telescope. The 1200 lines $/ \mathrm{mm}$ grating with a blaze angle of $13^{\circ}$ was used. The grating angle was set to $19^{\circ}$ to cover a wavelength range from $3950 \AA$ to $5250 \AA$. A $2048 \times 2048$ E2V CCD camera was used for the observations. The typical resolution of the spectra is $2.2 \AA$ and the dispersion $2.6 \AA$ per pixel. The reduction procedure was performed with the standard routines of the IRAF package. Examples of the reduced spectra are shown in Fig. 1. The final results of these observations will be published elsewhere (Fox Machado et al., in preparation).

The author acknowledges the financial support from the UNAM through grant PAPIIT IN104612 and from the IAU.

\section{References}

Aerts, C., Christensen-Dalsgaard, J., \& Kurtz, D. W. 2010, Asteroseismology (Springer Science+Business Media B. V.)

Borucki, W. J., Koch, D., Basri, G., et al. 2010, Science, 327, 977

Fox Machado, L., Alvarez, M., Michel, R., et al. 2010, New Astron., 15, 397

Latham, D. W., Brown, T. M., Monet, D. G., Everett, M., Esquerdo, G. A., \& Hergenrother, C. W. $2005, B A A S, 37,1340$

Molenda-Żakowicz, J., Latham, D. W., Catanzaro, G., Frasca, A., \& Quinn, S. N. 2011, MNRAS, 412,1210

Peña, J. H., Fox Machado, L., García, H., et al. 2011, Rev. Mexicana AyA, 47, 309

Uytterhoeven, K., Moya, A., Grigahcène, A., et al. 2011, Aछ A, 534, A125 